Check for updates

Cite this: RSC Adv., 2019, 9, 8355

\title{
Simple, selective detection and efficient removal of toxic lead and silver metal ions using Acid Red 94†
}

\author{
Ghada M. Kamel, ${ }^{\text {ab }}$ Marwa N. El-Nahass, ${ }^{a}$ Mohamed E. El-Khouly, (D)*bc \\ Tarek A. Fayed ${ }^{a}$ and Maged El-Kemary ${ }^{d}$
}

Toward the goal of detecting toxic elements and removing them from drinking water, we report herein the utilization of Acid Red 94 (AR94) in sensing the hazardous metal ions in water. Among the various examined metal ions $\left(\mathrm{Ag}^{+}, \mathrm{Pb}^{2+}, \mathrm{K}^{+}, \mathrm{Mn}^{2+}, \mathrm{Zn}^{2+}, \mathrm{La}^{3+}, \mathrm{Hg}^{2+}, \mathrm{Ca}^{2+}, \mathrm{Cd}^{2+}, \mathrm{Co}^{2+}\right.$, and $\left.\mathrm{Ni}^{2+}\right)$, the UV-visible absorption spectra showed high selectivity and sensitivity for toxic silver and lead metal ions in an aqueous solution. The observed absorption spectral changes and the rapid color changes confirm complex formation between AR94 and both $\mathrm{Ag}^{+}$and $\mathrm{Pb}^{2+}$ metal ions. The emission measurements showed the significant fluorescence quenching of the singlet excited state of AR94 in the presence of $\mathrm{Ag}^{+}$and $\mathrm{Pb}^{2+}$ metal ions suggesting the formation of an irradiative dye-metal complex under the prevailing experimental conditions. In order to remove the accumulated complexes of AR94 with silver metal ions, safe and harmless mesoporous titanium dioxide was utilized efficiently in removing the complexes with adsorption capacities of $91 \%$ at 30 minutes. These findings suggest a simple, fast and efficient method for both detecting silver in water, and removing the formed AR94-metal complexes in water. In addition, AR94 is shown to be a good sensor for the presence of $\mathrm{Ag}$ and $\mathrm{Pb}$ nanoparticles, NPs, in aqueous solution. The absorption and emission spectra of AR94 showed significant changes that may be rationalized by the strong electromagnetic coupling induced by NPs plasmonic effects. These findings render AR94 a sensitive and selective sensor and a visual indicator for the qualitative and quantitative detection of silver ions, lead ions and their nanoparticles.

Received 18th January 2019

Accepted 21st February 2019

DOI: $10.1039 /$ c9ra00464e

rsc.li/rsc-advances pressure, kidney damage, ${ }^{6}$ and diminished learning abilities in children. ${ }^{7}$ Although many people think that silver has no toxic side effects, silver toxicity has become a big problem in recent decades. $^{8}$

Recently, considerable efforts have been paid to developing highly selective and sensitive fluorescent probes for detection and removal of the toxic and radioactive species in drinking water. ${ }^{9-15}$ El-Nahass et al. developed a number of fluorescent chemosensors for metal ion detection in the environment. ${ }^{16-18}$ Also, J. Yoon et al. ${ }^{19}$ succeeded in designing fluorescent probes for the selective and sensitive detection of some metal ions, such as $\mathrm{Cu}^{2+}, \mathrm{Hg}^{2+}, \mathrm{Zn}^{2+}, \mathrm{Pb}^{2+}, \mathrm{Cd}^{2+}, \mathrm{Ag}^{+}$and $\mathrm{Au}^{3+}$, by introducing various ligands to core fluorophores, such as rhodamine, fluorescein, pyrene, anthracene, naphthalimide, coumarin, and BODIPY.

Herein, we report the selective and sensitive detection of silver and lead as hazard metal ions using a cheap and easily prepared Acid Red (AR94) dye. As is known, AR94 dye is a good probe for monitoring electrostatic interactions and hydrogen bonding interactions, ${ }^{20,21}$ and is widely used in photocatalytic reactions. To our knowledge, utilizing AR94 as a sensitive chemosensor is rarely reported in the literature. Furthermore, our main goal aimed at studying the ability to use Acid Red 94 dye as
${ }^{a}$ Department of Chemistry, Faculty of Science, Tanta University, Tanta, 31527, Egypt ${ }^{b}$ Department of Chemistry, Faculty of Science, Kafrelsheikh University, Kafr El-Sheikh 33516, Egypt

'Institute of Basic and Applied Sciences, Egypt-Japan University of Science and Technology (E-JUST), Alexandria, Egypt.E-mail: mohamed.elkhouly@ejust.edu.eg ${ }^{d}$ Institute of Nanoscience and Nanotechnology, Kafrelsheikh University, Kafr El-Sheikh 33516, Egypt

$\dagger$ Electronic supplementary information (ESI) available. See DOI: 10.1039/c9ra00464e 
a fluorescent chemosensor for the detection of metal ions such as lead and silver qualitatively (using naked eye detection) and quantitatively (using spectroscopic techniques at low concentration levels). Since the fabrication of nanoparticles (NPs) has attracted great attention in a wide range of environmental and technological applications in recent years, we have examined the electronic interactions of AR94 with silver and lead nanoparticles (NPs). As is well known, NPs exhibit different electrical, optical, magnetic, and chemical properties compared to their bulk counterparts, $\mathrm{Ag}^{+}$and $\mathrm{Pb}^{2+}$ metal ions. ${ }^{21-23}$ Finally, $\mathrm{TiO}_{2}$ mesoporous materials have been used as safe and harmless materials for the removal of the accumulated $\mathrm{AR94}-\mathrm{Ag}^{+}$complexes from the water under UV radiation in a fast and efficient way.

\section{Experimental section}

\subsection{Materials}

Acid Red dye (AR94) was purchased from TCI and used without any further purification. The metal salts as $\mathrm{AgNO}_{3}, \mathrm{~Pb}\left(\mathrm{NO}_{3}\right)_{2}$, $\mathrm{KCl}, \mathrm{MnCl}_{2} \cdot 4 \mathrm{H}_{2} \mathrm{O}, \mathrm{LaCl}_{3} \cdot 7 \mathrm{H}_{2} \mathrm{O}, \mathrm{ZnCl}_{2} \cdot 4 \mathrm{H}_{2} \mathrm{O}, \mathrm{HgCl}_{2} \cdot 2 \mathrm{H}_{2} \mathrm{O}$, $\mathrm{CaCl}_{2} \cdot 2 \mathrm{H}_{2} \mathrm{O}, \mathrm{CdNO}_{3} \cdot 4 \mathrm{H}_{2} \mathrm{O}, \mathrm{CoCl}_{2} \cdot 6 \mathrm{H}_{2} \mathrm{O}, \mathrm{NiCl}_{2} \cdot 6 \mathrm{H}_{2} \mathrm{O}, \mathrm{Pb}\left(\mathrm{CH}_{3}-\right.$ $\mathrm{COOH})_{2}$ and $\mathrm{Ag}_{2} \mathrm{SO}_{4}$ were obtained from Merck-Aldrich and used without purification. Trisodium citrate, sodium borohydride, Triton-X-100, cetyl trimethyl ammonium bromide (CTAB) and titanium butoxide were used as received from Aldrich.

\subsection{Synthesis}

2.2.1. Synthesis of colloidal AgNPs. Typically, $50 \mathrm{ml}$ of $0.001 \mathrm{M} \mathrm{AgNO}_{3}$ was heated till boiling. To this solution, $5 \mathrm{ml}$ of $1 \%$ trisodium citrate was added drop by drop and this was mixed vigorously. The solution was kept under continuous stirring at room temperature. ${ }^{24}$ Silver ions were reduced by trisodium citrate, which prevents the aggregation of the silver nanoparticles and stops them becoming larger. The concentration of the synthesized silver nanoparticles was calculated using the following equation; ${ }^{25}$

$$
N=\frac{\pi \rho D^{3}}{6 M} N_{\mathrm{A}}
$$

where $N$ is the number of atoms per nanoparticle, $D$ is the diameter of the AgNPs which was obtained from the TEM image $\left(18 \times 10^{-7} \mathrm{~cm}\right), \rho$ is the density of fcc $\mathrm{Ag}\left(10.5 \mathrm{~g} \mathrm{~cm}^{-3}\right),{ }^{25} \mathrm{M}$ is the atomic mass $\left(107.68 \mathrm{~g} \mathrm{~mol}^{-1}\right)$, and $N_{\mathrm{A}}$ is the Avogadro number.

$$
N=3.14 \times 10.5 \times\left(18 \times 10^{-7}\right)^{3} /(6 \times 107.68)=179251.59
$$

The molar concentration of the AgNPs solution, $C$, can be calculated using the following equation:

$$
C=\frac{N_{\text {total }}}{N V N_{\mathrm{A}}}
$$

where $N_{\text {total }}$ is the total number of $\mathrm{Ag}$ atoms (equivalent to the initial amount of its salt added to the reaction solution $=3.11 \times$ $10^{19}$ ), and $V$ is the volume of the reaction solution in litres = $0.05 \mathrm{ml}$.

$$
\begin{aligned}
C & =3.11 \times 10^{19} /\left(0.05 \times 6.023 \times 10^{23} \times 179251.59\right) \\
& =3.11 \times 10^{19} /\left(5.39 \times 10^{27}\right) \\
& =5.7 \times 10^{-9} \mathrm{M}
\end{aligned}
$$

2.2.2. Synthesis of PbNPs. $3.3 \mathrm{~g}$ of lead nitrate was added to $50 \mathrm{ml}$ deionized water, followed by the addition of Triton-X-100 $(0.5 \mathrm{ml})$ to make a $1 \%$ surfactant solution and this was stirred until a clear solution at room temperature was obtained. An aqueous solution of $\mathrm{NaBH}_{4}$ was then slowly injected into the solution. During this addition, the clear solution turned black, indicating the formation of $\mathrm{Pb}$ nanoparticles. The product was collected by centrifuge and washed several times with water and ethanol, then left to dry at $60{ }^{\circ} \mathrm{C}$ all night. ${ }^{26}$ The concentration of the prepared solution of PbNPs was found to be $0.02 \mathrm{M}$.

2.2.3. Synthesis of mesoporous $\mathrm{TiO}_{2}$. Mesoporous $\mathrm{TiO}_{2}$ was prepared via a sol-gel method. To synthesize titanium oxide nanoparticles, titanium butoxide $(10 \mathrm{ml})$ was added dropwise to a solution containing $75 \mathrm{ml}$ of deionized $\mathrm{H}_{2} \mathrm{O}$ and $2 \mathrm{~g}$ of CTAB under vigorous stirring at $80{ }^{\circ} \mathrm{C}$ for $24 \mathrm{~h}$. Then the resultant white gel suspension was filtered, washed several times with deionized water and ethanol, and then dried at $80{ }^{\circ} \mathrm{C}$ for $24 \mathrm{~h}$. The obtained sample was thermally treated and calcined at $450{ }^{\circ} \mathrm{C}$ for 4 hours using a Muffle furnace with a heating rate of $5{ }^{\circ} \mathrm{C} \min ^{-1}$.

2.2.4. Catalytic experiments. In order to get rid of the accumulated complex of AR94 with silver metal ions, the safe and harmless mesoporous titanium dioxide was utilized efficiently as an adsorbent for the removal of the formed complex. The catalytic degradation of the AR94- $\mathrm{Ag}^{+}$complex solution was performed by adding $0.01 \mathrm{~g}$ of mesoporous $\mathrm{TiO}_{2}$ to $50 \mathrm{ml}$ of $\mathrm{AR94}-\mathrm{Ag}^{+}$solution and increasing the radiation time using a UV lamp at $365 \mathrm{~nm}$. Before irradiation, the suspensions were stirred in a shaker water bath for a period of time to establish an adsorption/desorption equilibrium. The lamp was turned on 15 minutes prior to the reaction in order to attain a constant output of light intensity. The reaction mixtures in the flasks were shaken with simultaneous illumination and aliquots were drawn at regular intervals of time from 2 to 30 minutes, until equilibrium was reached. The absorbance of both elements of the complex after the adsorption process at equilibrium was estimated using steady-state absorption and emission techniques.

2.2.5. Instruments. The sensing process was investigated and confirmed by means of various spectroscopic techniques. UV-visible absorption spectra were recorded using a JASCO spectrophotometer (model V-780) in the UV-visible-near infrared region. Fluorescence spectra were recorded with a JASCO spectrofluorometer (model FP-8300). FT-IR spectra were recorded with JASCO FT/IR-4100 within the range of 400$4000 \mathrm{~cm}^{-1}$. The fast reactions of acid red dye with silver metal ions were detected using stopped-flow techniques. Small-angle powder X-ray diffraction (XRD) measurements were recorded at room temperature using a GNR, APD 2000 PRO step scans X-ray diffractometer, with $\mathrm{Cu} \mathrm{K} \alpha$ radiation $(40 \mathrm{kV}, 30 \mathrm{~mA})$, and a scanning range of $0-8^{\circ}(2 \theta)$, with a step of $0.02^{\circ}$. The pore shape and ordering of the mesostructured materials were 
examined and photographed using JEOL-JEM-100 SX transmission electron microscopy (TEM). Nanosecond transient absorption studies were performed using a nanosecond laser flash photolysis technique (LP980, Edinburgh Instruments, UK). The instrument was connected with a tunable laser source (NT342B-10, Ekspla).

\section{Results and discussion}

\subsection{Interaction of $\mathrm{AR94}$ and $\mathrm{Ag}^{+}$metal ions}

Fig. 1 shows the UV-visible absorption spectra of AR94 in the absence and presence of metal ions. As shown, the UV absorption spectrum of AR94 exhibited an absorption maximum at $\sim 520 \mathrm{~nm}$. Upon addition of the examined metal ions $\left(\mathrm{K}^{+}, \mathrm{Hg}^{2+}, \mathrm{Cd}^{2+}, \mathrm{Ni}^{2+}, \mathrm{Zn}^{2+}, \mathrm{Ca}^{2+}, \mathrm{Cu}^{2+}, \mathrm{La}^{+}\right.$, and $\left.\mathrm{Mn}^{2+}\right)$, the absorption spectrum of AR94 is kept almost the same suggesting no interactions between AR94 and these metal ions (ESI, Fig. S1-S4 $\dagger$ ). In the presence of $\mathrm{AgNO}_{3}$, it was interesting to see a rapid change of the color of AR94 from pink to violet color. In addition, the absorption spectrum of AR94 showed a considerable decrease of the intensity at $520 \mathrm{~nm}$, with an accompanying increase of a new band at longer wavelength $(\sim 600 \mathrm{~nm})$. This observation suggests the formation of a ground state complex (AR94-Ag). As seen from Fig. 2A, the absorption intensity of the formed complex increases with increasing the concentration of

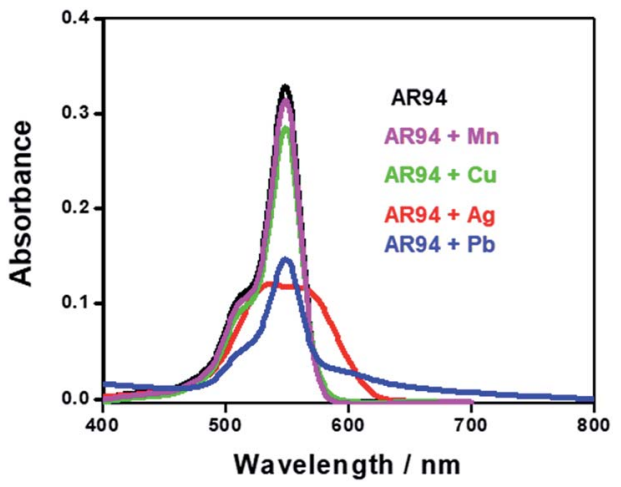

Fig. 1 UV-visible absorption spectra of AR94 in the absence and presence of lead and silver metal ions in water.
$\mathrm{Ag}$ metal ions. The existence of an isosbestic point at $580 \mathrm{~nm}$ confirms the formation of two species in equilibrium.

In a complementary experiment, the fluorescence spectra of AR94 in the absence and presence of $\mathrm{AgNO}_{3}$ were recorded in water, and are shown in Fig. 2B. Upon addition of different amounts of $\mathrm{Ag}^{+}$metal ions, it was found that the characteristic emission of the singlet state of AR94 at $\sim 560 \mathrm{~nm}$ is heavily quenched with a considerable small blue shift suggesting the formation of a non-emissive complex. Similar fluorescence quenching features were observed with the interaction of AR94 and silver sulfate. The calculated quenching rate constant of AR94- $\mathrm{Ag}_{2} \mathrm{SO}_{4}$ was found to be higher than that of AR94- $\mathrm{AgNO}_{3}$. This finding could be rationalized by the high negative charge density and high hydration energy of sulfate ions compared with nitrate ions.

Powerful nanosecond laser photolysis will also be utilized to examine the interaction between the excited AR94 and Ag ions in water. As is well known, nanosecond transient absorption spectroscopy is useful in measuring the kinetics of a population and decay of states following an initial excitation pulse. ${ }^{27,28}$ Fig. 3 shows the transient absorption spectrum of AR94 in an oxygenfree water solution obtained using excited $576 \mathrm{~nm}$ laser light. As shown, the transient spectrum exhibited absorption bands at 380 and $460 \mathrm{~nm}$. The decay rate of the triplet excited state at $460 \mathrm{~nm}$ was determined to be $5.71 \times 10^{4} \mathrm{~s}^{-1} \cdot{ }^{27-29}$ Upon addition of different concentrations of $\mathrm{AgNO}_{3}$, it was found that the transient spectra significantly decreased and the decay profiles of the triplet state at $460 \mathrm{~nm}$ were getting much faster. These findings confirm the interaction between the excited AR94 and the silver ions in an aqueous solution.

By measuring the kinetics of the fast reaction between AR94 and Ag metal ions, Fig. 4 shows the following observations: (i) the decay of the absorption band of AR94 is accompanied with the formation of an AR94-Ag complex, (ii) the reaction between Ag and AR94 is almost complete after 20 minutes, and (iii) the rates of complex formation increase with increasing the concentration of $\mathrm{Ag}$ metal ions. When increasing the concentration of added $\mathrm{AgNO}_{3}$ from $4.5 \times 10^{-6}$ to $1.1 \times 10^{-5} \mathrm{M}$, the reaction rates increase from $6.0 \times 10^{-3}$ to $3.4 \times 10^{-2} \mathrm{~min}^{-1}$, respectively.

The complex formation between AR94 and $\mathrm{Ag}^{+}$has been evidenced using an FTIR technique. As seen from Fig. 5, the free dye showed peaks at about 1547,1492 , and $1448 \mathrm{~cm}^{-1}$, which
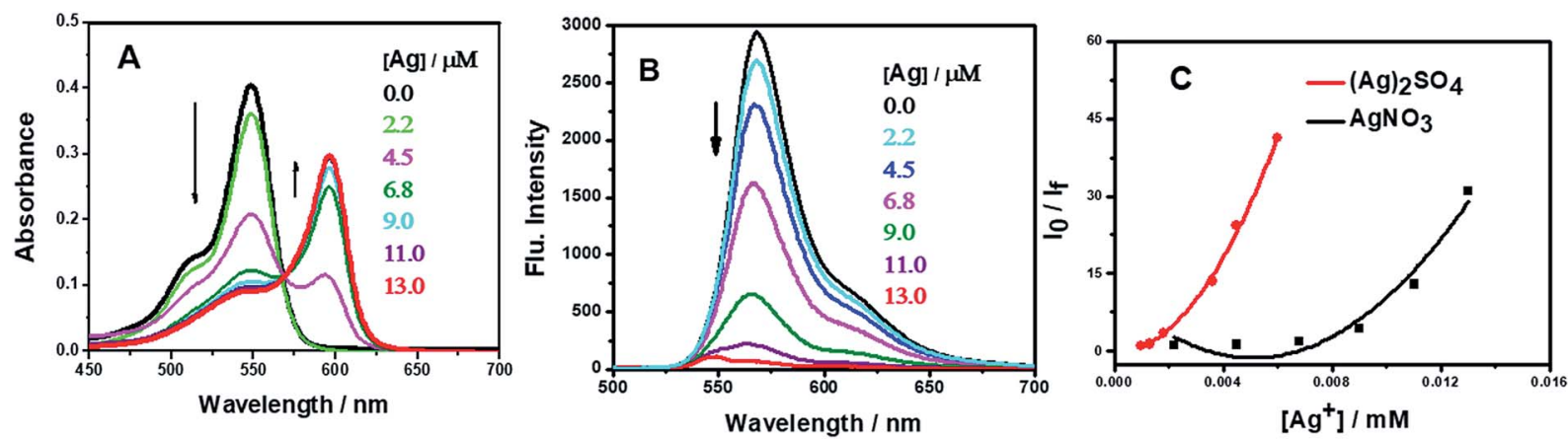

Fig. 2 (A) UV-visible absorption spectra and (B) emission spectra of AR94 with the addition of different concentrations of $\mathrm{AgNO}_{3} ; \lambda_{\text {ex }}=460 \mathrm{~nm}$. (C) Stern-Volmer plots for the fluorescence quenching of AR94 in the presence of Ag metal ions in water. 

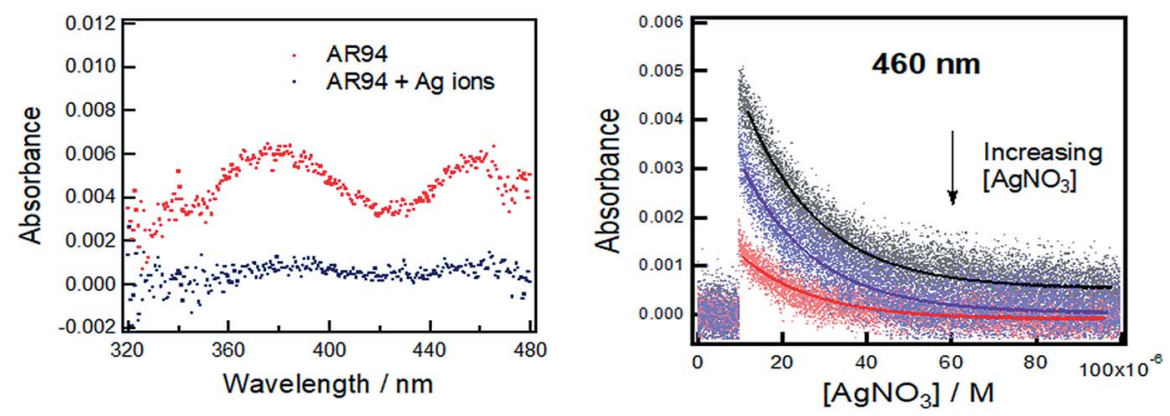

Fig. 3 (Left) Nanosecond transient absorption spectra of AR94 in the absence and presence of $\mathrm{AgNO}_{3}$ in water. (Right) Decay profiles of the triplet AR94 at $460 \mathrm{~nm}$ with increasing $\left[\mathrm{AgNO}_{3}\right] ; \lambda_{\mathrm{ex}}=560 \mathrm{~nm}$.
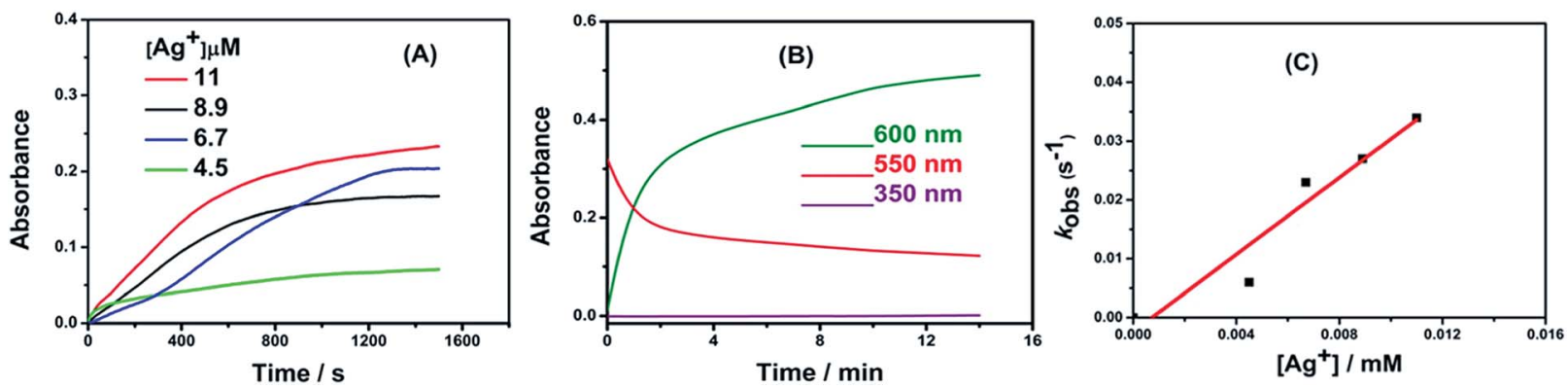

Fig. 4 (A) Kinetics measurements of the rapid interaction of AR94 dye with $\mathrm{Ag}^{+}$metal ions. (B) Rate of reaction between dye and $\mathrm{Ag}^{+}$metal ions measured at different wavelengths. (C) Relation between $k_{\text {obs }}$ and concentration of silver nanoparticles.

are ascribed to the characteristic absorption of the vibration of the aromatic ring frame of AR94. A peak at $1235 \mathrm{~cm}^{-1}$ was recorded and corresponds to $\mathrm{C}-\mathrm{O}-\mathrm{C}$ groups. The observed peaks at 1613 and $1339 \mathrm{~cm}^{-1}$ could be attributed to the asymmetric and symmetric stretching vibrations of $\mathrm{COO}^{-}$, respectively. The characteristic absorption bands of $\mathrm{C}-\mathrm{Cl}$ stretching vibrations and $\mathrm{Na}-\mathrm{O}$ bonds were observed at 759 and $1020 \mathrm{~cm}^{-1}$, respectively. When turning to the AR94- $\mathrm{Ag}$ complex, new peak was recorded at $1110 \mathrm{~cm}^{-1}$ that can be assigned to the characteristic $\mathrm{Ag}-\mathrm{O}$ bond, confirming the formation of an AR94-Ag complex (Scheme 1)..$^{30,31}$

\subsection{Interaction of $\mathbf{A R 9 4}$ and $\mathbf{P b}^{2+}$ metal ions}

The selectivity of AR94 for $\mathrm{Pb}^{2+}$ metal ions has also been investigated by measuring the absorbance and emission spectra

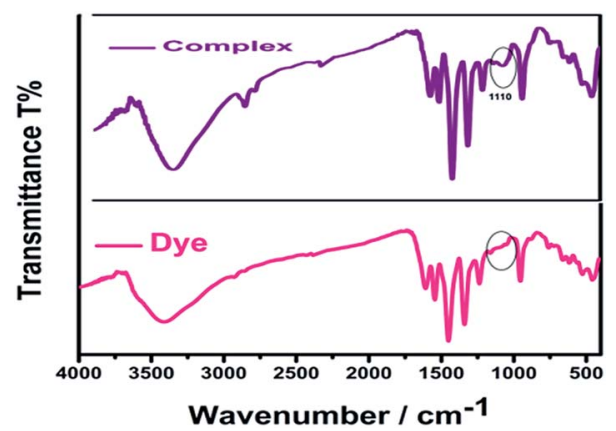

Fig. 5 FTIR spectra of AR94 (lower panel) and the AR94-Ag complex (upper panel).
(Fig. 6). Upon adding different concentrations of $\mathrm{Pb}^{2+}$ metal ions, the UV-visible absorption spectra of AR94 showed a significant decrease in the absorption intensity, accompanied with the formation of an isosbestic point at $580 \mathrm{~nm}$ and a new peak around $600 \mathrm{~nm}$. Additionally, an observable change in the color from pink to violet by the addition of $\mathrm{Pb}^{2+}$ metal ions has been noticed. These observations suggest the formation of a fast ground state complex (AR94- $\mathrm{Pb}^{2+}$ ). Similarly, the fluorescence intensity of the singlet AR94 dye showed a significant quenching was observed with the addition of various amounts of $\mathrm{Pb}^{2+}$ metal ions. The quenching follows the Stern-Volmer relation $\left(I_{\mathrm{o}} / I=1+K_{\mathrm{SV}}[\mathrm{Q}]\right)$, where $I_{\mathrm{o}}$ and $I$ are the emission intensities of AR94 in the absence and presence of a quencher of concentration [Q], respectively, and $K_{\mathrm{SV}}$ is the Stern-Volmer quenching constant. The Stern-Volmer plots show an upward curvature, especially at higher concentrations, confirming the static quenching (Fig. 6). This higher quenching of AR94 in the presence of lead acetate was found to be higher than that of AR94/lead nitrate, which may be explained by the high $\mathrm{p} K_{\mathrm{a}}$ and high protonation energy of acetate anions compared to nitrate anions. ${ }^{32}$ It is most likely that the high selectivity of Acid Red 94 towards $\mathrm{Ag}^{+}$and $\mathrm{Pb}^{2+}$ metal ions may be related to the their appropriate ionic radii (115 and $119 \mathrm{pm}$ for $\mathrm{Ag}$ and $\mathrm{Pb}$, respectively). ${ }^{33}$ In addition, the formation of the complex between AR94 and $\mathrm{Pb}^{2+}$ has been confirmed by FTIR analysis. As seen from Fig. 7, a new band centered at $716 \mathrm{~cm}^{-1}$ has been observed that can be attributed to $\mathrm{Pb}-\mathrm{O}$ bond vibrations and confirms the formation of AR94-Pb complexes. ${ }^{34}$ 
<smiles>O=C(O)c1c(Cl)c(Cl)c(Cl)c(Cl)c1-c1c2cc(I)c(=O)c(I)c-2oc2c(I)c(O)c(I)cc12</smiles><smiles>O=C(O)c1c(Cl)c(Cl)c(Cl)c(Cl)c1-c1c2cc(I)c(=O)c(O)c-2oc2c(I)c(O[Na])c(I)cc12</smiles>

Scheme 1 Suggested binding mode of $\mathrm{AR9} 9$ with $\mathrm{AgNO}_{3}$
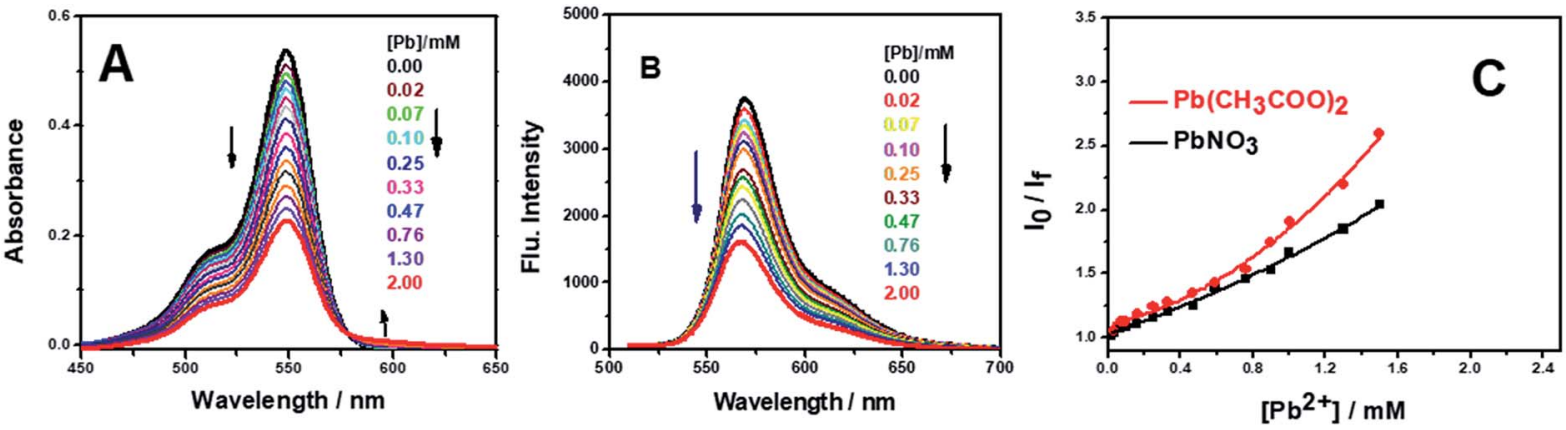

Fig. 6 (A) UV-visible absorption spectra and (B) emission spectra of AR94 in the absence and presence of $\mathrm{Pb}^{2+}$ metal ions in water. (C) SternVolmer plots for the fluorescence quenching of the singlet AR94 in the presence of lead metal nitrate and lead acetate in water.

\subsection{Interaction of AR94 with silver and lead nanoparticles}

3.3.1. Characterizations of silver and lead nanoparticles. Fig. 8A shows the X-ray diffraction pattern of the synthesized PbNPs. The XRD peaks correspond to the face-centered cubic crystal structure of $\mathrm{Pb}$ standard diffraction (JCPDS card number: 02-0799). ${ }^{14}$ The $(2 \theta)$ peaks positioned for $\mathrm{Pb}$ at $30.34,37.33,52.32$ and $65.36^{\circ}$ correspond to the reflection lattice planes of (111), (200), (220) and (222), respectively. From the Scherrer equation, it seems that the size of the PbNPs is about $45 \mathrm{~nm}$. Fig. 8B shows the absorption spectra of the synthesized PbNPs and AgNPs. As can be seen, the UV absorption spectra for PbNPs and AgNPs exhibit an intense peak at $230 \mathrm{~nm}$ and $415 \mathrm{~nm}$ respectively, confirming the formation of NPs. Fig. 8C shows a scanning electron microscope image which confirms the spherical shape of PbNPs and their purity has been confirmed by the EDX picture shown in Fig. 8D. It was found that PbNPs are $92.49 \%$ of the

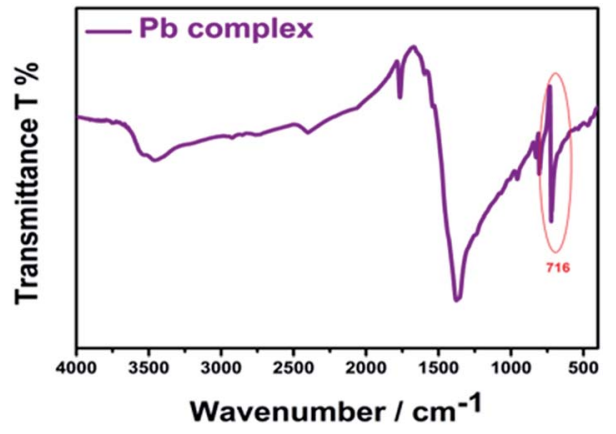

Fig. 7 FTIR spectra of the AR94-Pb complex. sample and the other $7.5 \%$ was the carbon grade of the test. TEM images showed the spherical shape of AgNPs with an average size of about $18 \mathrm{~nm}$ (Fig. 8E), while the size of the PbNPs was determined to be in the range of 18 to $25 \mathrm{~nm}$ with the effect of the surfactant Triton-X-100 (Fig. 8F).

3.3.2. Effect of AR94 with AgNPs and PbNPs. Fig. 9 shows the absorption and emission spectra of AR94 in the absence and presence of different concentrations $\left(0.25-4.7 \times 10^{-10} \mathrm{M}\right.$ and 0.5-5.3 mM) of AgNPs and PbNPs, respectively. Upon increasing the concentrations of AgNPs, the absorption spectra decrease gradually accompanied by the formation of a new absorption band of AR94-AgNPs in the visible region at $580 \mathrm{~nm}$. Additionally, two isosbestic points at 470 and $570 \mathrm{~nm}$ were observed with an instantaneous color change to violet. Similar absorption features were recorded for the interaction of AR94-PbNPs, where the absorption band of AR94 decreases accompanied by the complex formation in the range of 600-800 $\mathrm{nm}$. Two isosbestic points at 535 and $560 \mathrm{~nm}$ were recorded, along with a quick color change from pink to violet, confirming the complex formation. Compared to the corresponding absorption spectra measured in the case of $\mathrm{Pb}^{2+}$ metal ions, dramatic changes in the shape and position of the formed complex have been noticed, which may be due to strong electromagnetic coupling between the metal and the dye, induced by PbNPs plasmonic effects.

However, as the concentration of $\mathrm{Ag}^{+} / \mathrm{Pb}^{2+}$ metal ions and/or NP quencher increases, the positions of the fluorescence bands do not change despite the substantial decrease in the fluorescence intensities. This indicates the absence of significant molecular interactions under the prevailing experimental 

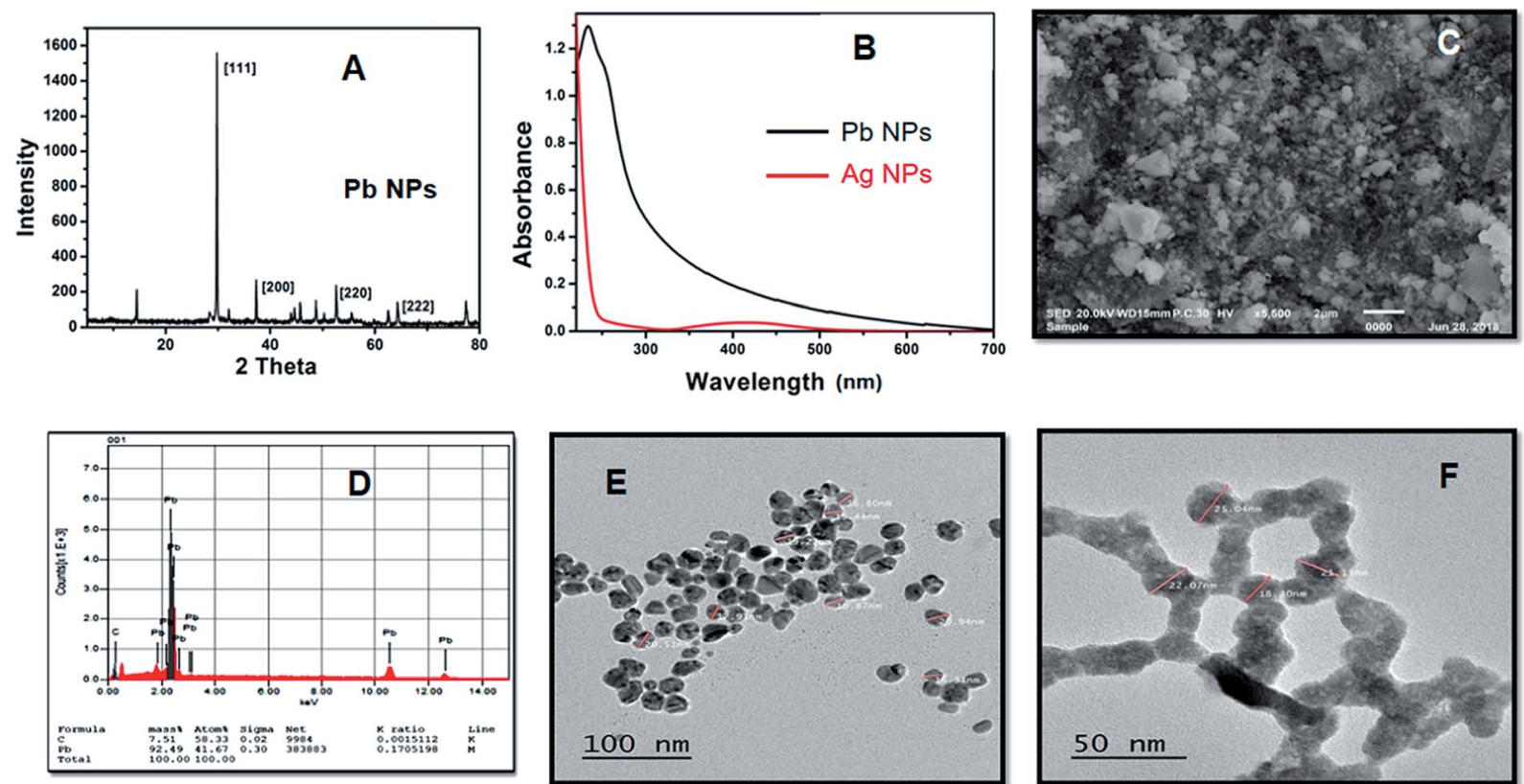

Fig. 8 (A) XRD pattern of lead NPs, (B) UV-visible absorption spectra of lead and silver NPs, (C) SEM image of lead NPs, (D) EDX of lead NPs, (E) TEM image for silver NPs, and (F) TEM image of lead NPs.

conditions. Generally, from the notable spectral and fast color changes of the investigated acid dye upon addition of the synthesized NPs, we conclude that the investigated dye can potentially become a sensitive and selective sensor for the qualitative and quantitative detection of the used metal ions and could be used as a visual indicator.

Despite the importance of AR94 acting as a sensor for metal ion detection, there must be a way to get rid of the accumulated complex (AR94-Ag), which will negatively affect the
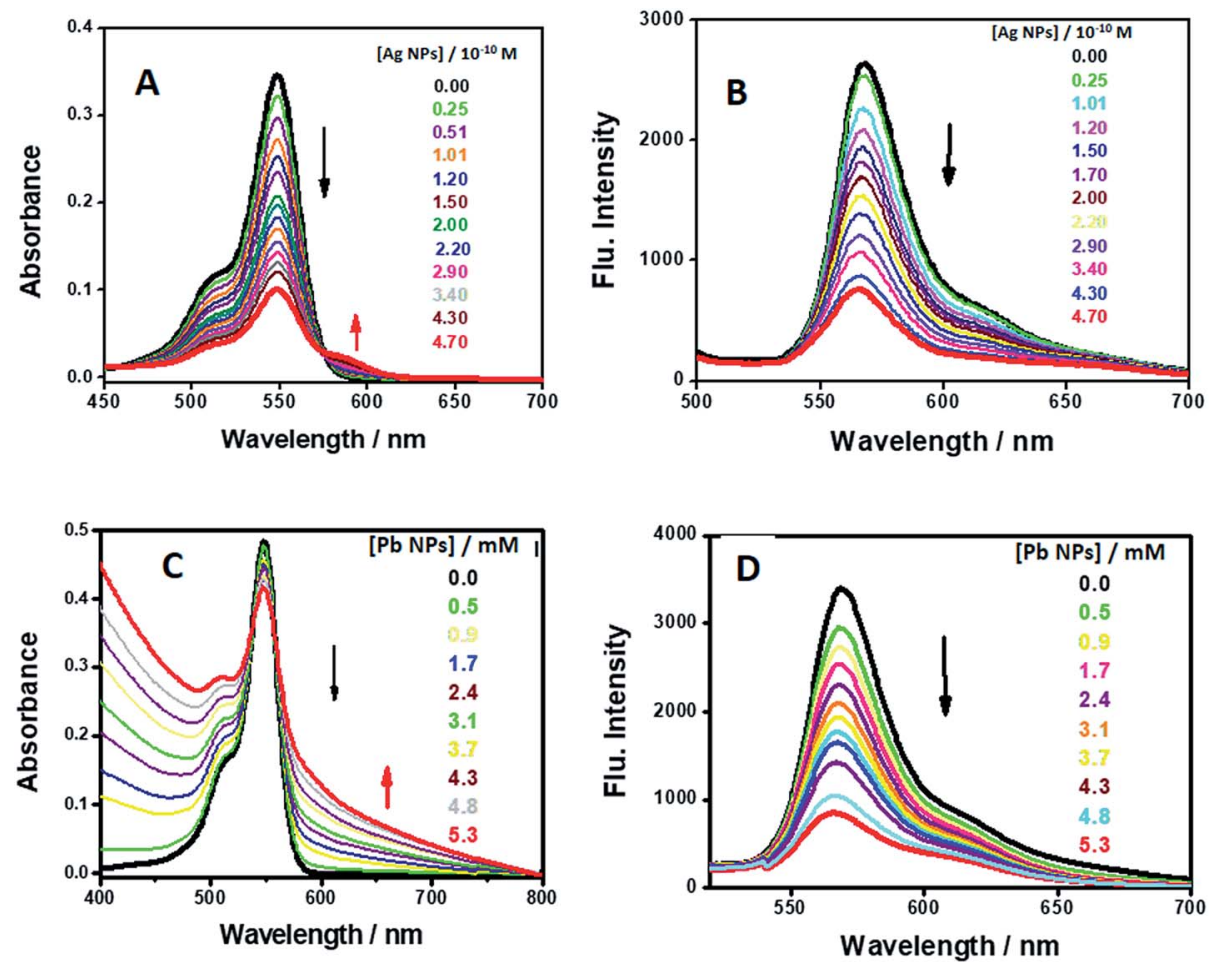

Fig. 9 UV-visible absorption spectra and emission spectra of AR94 in the absence and presence of different concentrations of the synthesized AgNPs (A and $\mathrm{B})$ and PbNPs (C and $\mathrm{D})$. 

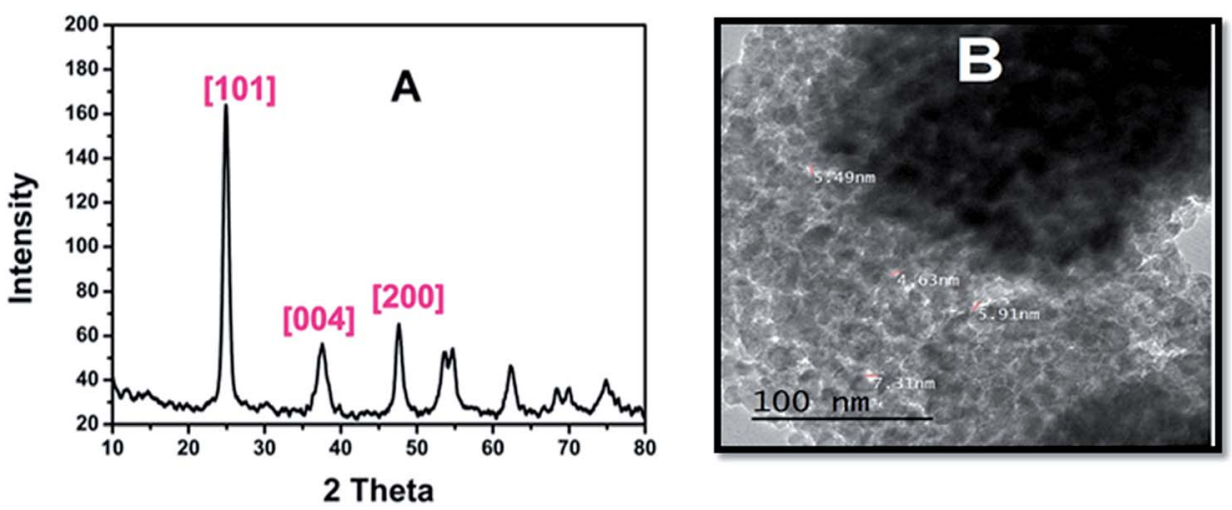

Fig. 10 XRD pattern (A) and TEM image (B) of mesoporous $\mathrm{TiO}_{2}$.
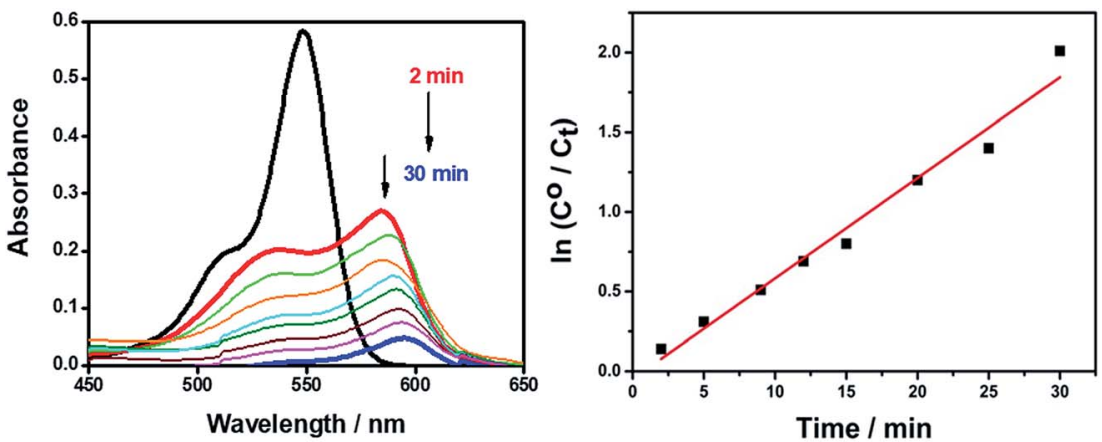

Fig. 11 UV-visible absorption spectra of removing $\mathrm{AR94}-\mathrm{Ag}^{+}$complexes using mesoporous $\mathrm{TiO}_{2}$ under continuous UV radiation.

water. Therefore, we have synthesized mesoporous titanium dioxide, which is a safe and harmless material, in order to remove these complexes. Fig. 10A shows a representative XRD pattern of mesoporous $\mathrm{TiO}_{2}$. As can be seen, all peaks can be indexed to the pure structure of $\mathrm{TiO}_{2}$ without any impurities and the broadening of the peaks is due to the small size of the nanoparticles. The $(2 \theta)$ peaks positioned for $\mathrm{TiO}_{2}$ at 25.02, 37.05 , and 47.82 , correspond to the reflection lattice planes of (101), (004), and (200), respectively. ${ }^{35}$ Fig. 10B shows a TEM image that further reveals the porous nature of the materials. The pores in the mesoporous spheres $(\sim 5 \mathrm{~nm})$ are clearly observed. The mesoporous spheres are composed of a lot of interconnected nanoparticles with sizes around $10 \mathrm{~nm}$, which is compatible with results calculated theoretically using Scherrer's equation.

Fig. 11 shows the degradation of the AR94-Ag complex solution on adding $0.01 \mathrm{~g}$ of mesoporous $\mathrm{TiO}_{2}$ with increasing the radiation time using UV lamp at $365 \mathrm{~nm}$. Before irradiation, the suspensions were stirred in a shaker water bath for a period of time to establish the equilibrium. As can be seen, the absorption of AR94-Ag complex decreases gradually with time. This can be rationalized by the adsorption of the complex on the surface of mesoporous $\mathrm{TiO}_{2}$. The removal efficiency was determined to be $91 \%$ at $30 \mathrm{~min}$. The high and fast efficiency of using mesoporous $\mathrm{TiO}_{2}$ to remove the free AR94 could be rationalized by the generated electrons and holes in the conduction band and valence band of $\mathrm{TiO}_{2}$, generated by UV irradiation, respectively. The electrons in the conduction band can react with oxygen to produce superoxide radical anions $\mathrm{O}_{2}{ }^{-} \cdot{ }^{36,37}$ These superoxide radical anions further produce hydroxyl radicals as well as reacting with a proton to form hydroperoxyl radicals $\left(\mathrm{HO}_{2}{ }^{\circ}\right)$. The positive hole can oxidize hydroxide ions (or water molecules) adsorbed on the surface of $\mathrm{TiO}_{2}$ particles to produce hydroxyl radicals. ${ }^{38}$ The generated radicals are usually reacting with AR94 followed by the formation of several intermediates, and it is most likely that it is finally mineralized into carbon dioxide and water.

The observed rate constants for the photocatalytic degradation of this complex with mesoporous $\mathrm{TiO}_{2}$ were determined using the relation: $\ln \left(C / C^{0}\right)=-k_{\text {obs }} t$, where $C^{0}\left(\mathrm{mg} \mathrm{L}^{-1}\right)$ is the initial concentration and $k_{\text {obs }}$ depends on the initial concentration $\left(C^{0}\right) \cdot{ }^{39,40}$ From the linear plot of $\ln \left(C / C^{0}\right)$ with irradiation time, the degradation rate $(k)$ of the complex in the presence of mesoporous $\mathrm{TiO}_{2}$ was found to be $0.063 \mathrm{~min}$.

\section{Conclusion}

Herein, we report the utilization of the commercial AR94 dye as a sensor for the detection of toxic lead and silver metal ions in water. The interaction between AR94 and both metal ions was proved quantitatively by the absorption and fluorescence spectral changes, and qualitatively by the rapid color change from a pink color (for AR94) to a violet color (for the AR94-Ag complex). The removal of the accumulated complex (AR94-Ag) 
was successfully performed using safe and harmless mesoporous titanium dioxide with an adsorption capacity of $91 \%$ at $30 \mathrm{~min}$. The high and fast efficiency of mesoporous $\mathrm{TiO}_{2}$ was due the generation of radicals which usually react with the free dye particles followed by the formation of several intermediates which are finally mineralized into carbon dioxide and water. So, the removal process in water was easy and safe as the complex is adsorbed on the mesoporous $\mathrm{TiO}_{2}$. Furthermore, the interactions between AR94 and the fabricated $\mathrm{Ag}$ and/or $\mathrm{Pb}$ nanoparticles have been examined. Compared to the corresponding UV-visible absorption spectra measured in the case of $\mathrm{Ag}^{+}$or $\mathrm{Pb}^{2+}$ metal ions, dramatic changes in the shape and position of the formed complex have been noticed, which may be due to strong electromagnetic coupling between the metal and the dye, induced by NPs plasmonic effects. From the notable spectral changes and fast color changes of AR94 with the addition of silver, lead and their nanoparticles, we conclude that AR94 can potentially become a sensitive and selective sensor for the qualitative and quantitative detection of the used metal ions and could be used a visual indicator.

\section{Conflicts of interest}

The authors declare no conflict of interest.

\section{Acknowledgements}

This work was financially supported by the Science and Technology Development Fund (STDF), Egypt, Grant Numbers 5537 and 12436.

\section{References}

1 C. H. Lee, H. Miyaji, D. W. Yoon and J. L. Sessler, Chem. Commun., 2008, 1, 24-34.

2 S. M. Mousavi, S. A. Hashemi, M. Zarei, A. M. Amani and A. Babapoor, Med. Chem., 2018, 8, 205-217.

3 K. Sardar, S. Ali, S. Hameed, S. Afzal, S. Fatima, M. B. Shakoor, S. A. Bharwana and H. M. Tauqeer, Greener J. Environ. Manag., 2013, 2, 172-179.

4 H. T. Ratte, Environ. Toxicol. Chem., 1999, 18, 89-108.

5 R. Baran, D. A. R. de Berker, M. Holzberg and L. Thomas, Baran and Dawber's Diseases of the Nails and their Management, John Wiley \& Sons, 2012, pp. 1-417.

6 E. B. Ekong, B. G. Jaar and W. M. Weaver, Kidney Int., 2006, 70, 2074-2084.

7 W. L. Harold and W. S. Barton, Int. J. Environ. Health Res., 2016, 26, 5-6.

8 W. L. Roper, Toxicological profile for silver, Agency for Toxic Substances and Disease Registry, U.S. Public health Service, 1990.

9 N. M. El-Shafai, M. E. El-Khouly, M. El-Kemary, M. S. Ramadan, A. S. Derbalah and M. S. Masoud, J. Ind. Eng. Chem., 2019, 69, 315-323.

10 A. F. Tag El-Din, E. A. Elshehy and M. E. El-Khouly, J. Environ. Chem. Eng., 2018, 6, 5845-5854.
11 A. F. Tag El-Din, M. E. El-Khouly, E. Elshehy, A. A. Atia and W. A. El-Said, Microporous Mesoporous Mater., 2018, 265, 211-218.

12 X. F. Zhang, Z. G. Liu, W. Shen and S. Gurunathan, Int. J. Mol. Sci., 2016, 17, 1-34.

13 K. D. Datkhile, P. P. Durgawale and M. N. Patil, Biomed. Pharmacol. J., 2017, 10, 337-344.

14 V. Marassi, L. Di Cristo, S. G. J. Smith, S. Ortelli, M. Blosi, A. L. Costa, P. Reschiglian, Y. Volkov and A. Prina-Mello, $R$. Soc. Open Sci., 2018, 5, 1-21.

15 T. Sadhasivam, K. Dhanabalan, S. H. Roh, S. C. Kim, D. Jeon, J. E. Jin, J. Shim and H.-Y. Jung, J. Mater. Sci., 2017, 28, 56695674.

16 M. N. El-Nahass and T. A. Fayed, Appl. Organomet. Chem., 2017, 31, 1-13.

17 M. N. El-Nahass, M. Shaaban, T. Fayed and F. Hassan, Int. J. Chem. Appl. Biol. Sci., 2014, 1, 1-40.

18 M. N. El-Nahaa, T. A. Fayed, M. H. Shaaban and F. M. Hassan, Sens. Actuators, B, 2015, 210, 56-68.

19 Y. Jeong and J. Yoon, Inorg. Chim. Acta, 2012, 381, 2-14.

20 D. Xu and D. C. Neckers, J. Photochem. Photobiol., A, 1987, 40, 361-370.

21 M. Blosi, S. Ortelli, A. I. Costa, M. Dondi, A. Lolli, S. Andreoli, P. Benito and S. Albonetti, Materials, 2016, 9, 1-25.

22 A. Yadav, L. Zhong, J. Sun, L. Jiang, G. J. Cheng and L. Chi, Nano Convergence, 2017, 4, 1-8.

23 J. Jeevanandam, A. Barhoum, Y. S. Chan, A. Dufresne and M. K. Danquah, Beilstein J. Nanotechnol., 2018, 9, 10501074.

24 S. Asta, P. Judita and T. Sigitas, Mater. Sci., 2009, 15, 2127.

25 X. Lui, M. Atwater, Q. Wang and J. Huo, Colloids Surf., B, 2007, 58, 3-7.

26 K. Kannan, G. Muthuraman and S. Moon, Mater. Lett., 2014, 123, 19-22.

27 M. El-Khouly, P. Padmawar, Y. Araki, S. Verma, L. Y. Chiang and O. Ito, J. Phys. Chem. A, 2006, 110, 884-891.

28 M. E. El-Khouly, S. Gaddem, G. R. Deviprasad, M. Fujitsuka, O. Ito and F. D'Souza, J. Porphyrins Phthalocyanines, 2003, 7, $1-7$.

29 M. E. El-Khouly, E. El-Mohsnawy and S. Fukuzumi, J. Photochem. Photobiol., C, 2017, 13, 36-83.

30 Z. An, W. Zhang, H. Shi and J. He, J. Catal., 2006, 241, 319327.

31 H. Yang, S. Li, X. Wang, F. Zhang, X. Zhong, Z. Dong and J. Ma, J. Mol. Catal. A: Chem., 2012, 364, 404-410.

32 P. Bühlmann, E. Pretsch and E. Bakker, Chem. Rev., 1997, 97, 3083-3132.

33 R. D. Shannon, Acta Crystallogr., Sect. A: Cryst. Phys., Diffr., Theor. Gen. Crystallogr., 1976, 32, 751-767.

34 M. R. Ahsan, M. A. Uddin and M. G. Mortuza, Indian J. Pure Appl. Phys., 2005, 43, 89-99.

35 C. H. Kwon, H. M. Shin, J. H. Kim and W. S. Choi, Mater. Chem. Phys., 2004, 86, 78-82.

36 H. A. Le, L. T. Linh and S. Chin, Powder Technol., 2012, 225, 167-175. 
37 G. H. Wang, F. Wu, X. Zhang, M. Luo and N. Deng, J. Hazard. Mater., 2006, 133, 85-91.

38 R. J. Wu, C. C. Chen and C. S. Lu, Desalination, 2010, 250, 869-875.
39 E. Bizani, K. Fytianos, I. Poulios and V. Tsiridis, J. Hazard. Mater., 2006, 136, 85-94.

40 M. Sundararajan, L. J. Kennedya, P. Nithy, J. J. Vijaya and M. Bououdin, J. Phys. Chem. Solids, 2017, 108, 61-75. 